S. Chen

\title{
Emittance Growth Saturation Effect in Synchrotron Machines Due to Point-Like Perturbations
}

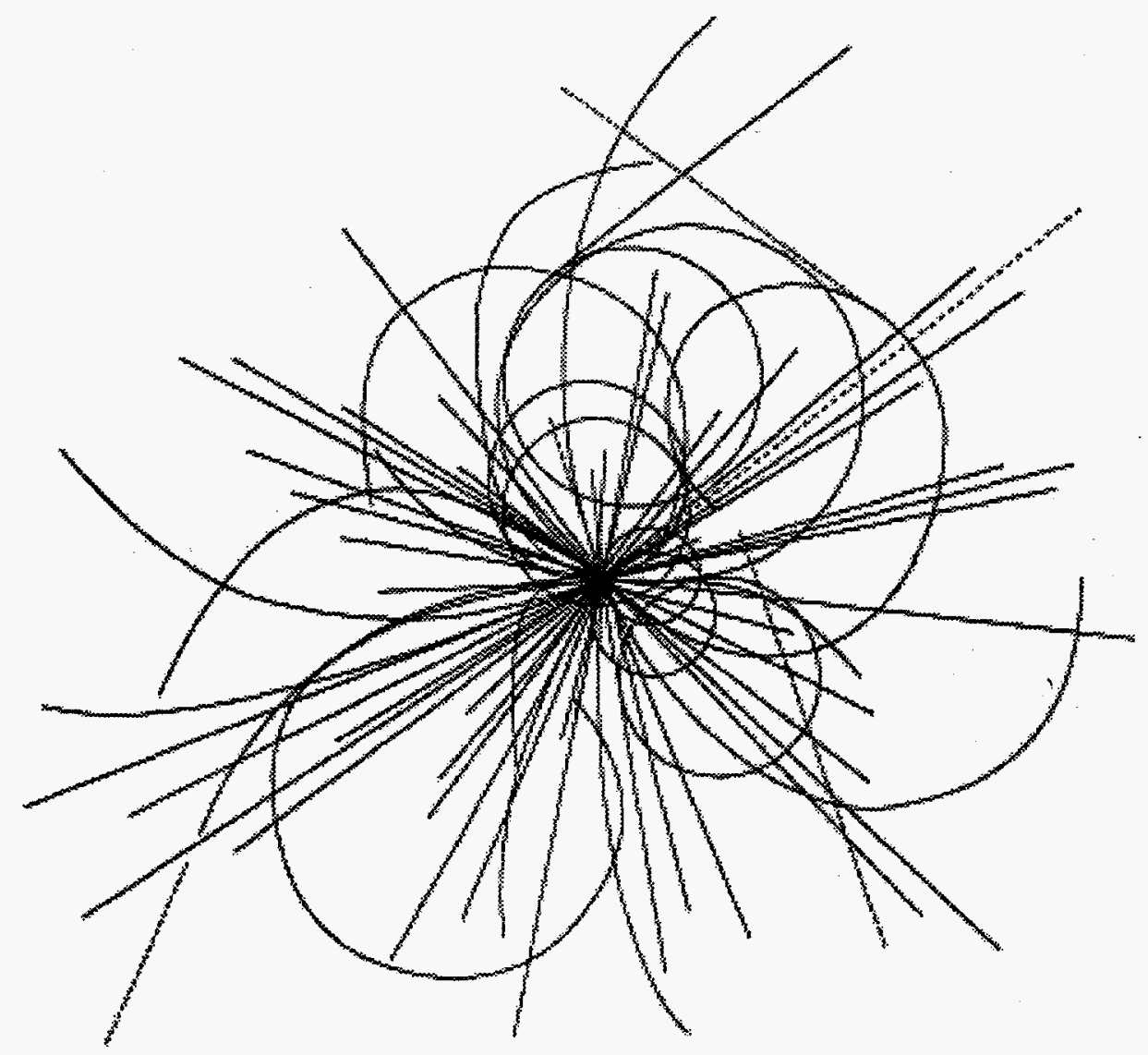

\section{Superconducting Super Collider Laboratory}

APPROVED FOR RELEASE OR PUBLICATION - ORR. PATENT GROUP BY...8. 


\section{Disclaimer Notice}

This report was prepared as an account of work sponsored by an agency of the United States Government. Neither the United States Government or any agency thereot, nor any of their employees, makes any warranty, express or implied, or assumes any legal liability or responsibility for the accuracy, completeness, or usefulness of any intormation, apparatus, product, or process disclosed, or represents that its use would not infringe privately owned rights. Reference herein to any specific commercial product, process, or service by trade name, trademark, manufacturer, or otherwise, does not necessarily constitute or imply its endorsement, recommendation, or favoring by the United States Government or any agency thereof. The views and opinions of authors expressed herein do not necessarily state or reflect those of the United States Govemment or any agency thereot.

Superconducting Super Collider Laboratory is an equal opportunity employer. 


\section{DISCLAIMER}

Portions of this document may be illegible in electronic image products. Images are produced from the best available original document. 


\title{
Emittance Growth Saturation Effect in Synchrotron Machines Due to Point-Like Perturbations*
}

\author{
G. López and S. Chen \\ Superconducting Super Collider Laboratory ${ }^{\dagger}$ \\ 2550 Beckleymeade Avenue \\ Dallas, Texas 75237
}

November 1993

\footnotetext{
" Submitted to PHYSICA $A$.

† Operated by the Universities Research Association, Inc., for the U.S. Department of Energy under Contract No. DE-AC35-89ER40486.
} 


\begin{abstract}
Analytical estimation of the transverse emittance growth due to a point-like perturbation is performed for a proton synchrotron machine. This emittance growth is caused by the tune spread within the bunch. However, the emittance growth suffers a saturation effect caused by the same tune spread. Computer simulations on the emittance growth due to resistive wall instabilities and feedback systems verify qualitatively this emittance growth saturation effect. These simulations were accomplished in the Medium Energy Booster of the Superconducting Super Collider using the TADIMMI computer code.
\end{abstract}

\title{
I. INTRODUCTION
}

Future high-luminosity synchrotron machines such as the Large Hadron Collider (LHC) [1], the Superconducting Super Collider (SSC) [2], $B$-factories, and a new generation of synchrotron light sources are required to store a large quantity of particle bunches for a long time. As a result, multibunch instabilities due to resistive wall impedance of the rf cavities are likely to appear in the beam. To control the coherent oscillations of these instabilities, a feedback system may be required. However, even if these oscillations are controlled, external noises [3] such as ground vibrations, power supply ripples, running fluid inside the magnets, and feedback noise [4] may cause emittance growth in the beam. This represents a threat mainly to the ability of proton synchrotron collider machines to make new discoveries, because the decrease of luminosity in the machine lessens the likelihood of collision. For electron synchrotron machines, the radiation damping helps to stabilize the emittance of the beam [5]. Initial estimations of the emittance growth in proton machines [3,4] make use of some type of approximation in the random process (noise). In this paper, those approximations will be avoided, and the approach given in Reference 4 will be followed. In addition, the synchrotron radiation damping effect will be neglected, since the emittance growth on the proton machines is our main interest here. 


\section{ANALYTICAL ESTIMATION}

Assume an ideal linear synchrotron machine having $M$ bunches, and each bunch having $N_{B}$ charged particles. These bunches are guided and shaped by the electromagnetic field of the devices in the machine (magnets, rf system, etc.). Assume also that these bunches suffer additional perturbations described by the function $F(s)$, and that different particles in the bunch have different tunes. The equation of motion of the centroid of the bunch and that of one specific particle within the bunch are given by [6]

$$
\frac{d^{2} \eta_{c}}{d \phi^{2}}+\nu_{o}^{2} \eta_{c}=f(\phi)
$$

and

$$
\frac{d^{2} \eta}{d \phi^{2}}+\nu^{2} \eta=f(\phi)
$$

where $\eta$ and $\phi$ are new variables defined as

$$
\eta(s)=X(s) / \sqrt{\beta(s)}
$$

and

$$
\phi(s)=\frac{1}{\nu} \int_{0}^{s} \frac{\sigma}{\beta(\sigma)},
$$

$f$ is the function $f(\phi)=\nu^{2} \beta^{3 / 2}(s(\phi)) F(s(\phi)), X$ is the amplitude of oscillation of the particle, $s$ is the longitudinal variable, $\beta$ is a Courant-Snyder variable, $\nu_{o}$ is the centroid tune of the bunch, and $\nu$ is the particle tune. Using the relative variable $q$,

$$
q(\phi)=\eta-\eta_{c}
$$

the system of Eqs. (1a) and (1b) is transformed to (1a), and

$$
\frac{d^{2} q}{d \phi^{2}}+\nu^{2} q=\left(\nu_{o}^{2}-\nu^{2}\right) \eta_{c}(\phi)
$$

The solution of Eq. (1a) is well-known [7]:

$$
\eta_{c}(\phi)=\eta_{c h}(\phi)+\frac{1}{\nu_{o}} \int^{\phi} f\left(\phi^{\prime}\right) \sin \nu_{o}\left(\phi-\phi^{\prime}\right) d \phi^{\prime},
$$


where $\eta_{c h}$ is the homogeneous solution of Eq. (1a). Using Eq. (6) in Eq. (5), the solution of Eq. (5) can be written as

$$
\begin{aligned}
q(\phi)= & q_{h}(\phi)+\frac{\nu_{o}^{2}-\nu^{2}}{\nu} \int^{\phi} \eta_{c h}\left(\phi^{\prime}\right) \sin \nu\left(\phi-\phi^{\prime}\right) d \phi^{\prime} \\
& +\frac{\nu_{o}^{2}-\nu^{2}}{\nu_{o} \nu} \int^{\phi} \sin \nu\left(\phi-\phi^{\prime}\right) d \phi^{\prime} \int^{\phi^{\prime}} f(\xi) \sin \nu_{o}\left(\phi^{\prime}-\xi\right) d \xi
\end{aligned}
$$

where $q_{h}$ is the homogeneous solution of Eq. (5). The conjugated variable of $q, p=d q / d \phi$, is given by the following expression:

$$
\begin{aligned}
p(\phi)= & p_{h}(\phi)+\left(\nu_{o}^{2}-\nu^{2}\right) \int^{\phi} \eta_{c h}\left(\phi^{\prime}\right) \cos \nu\left(\phi-\phi^{\prime}\right) d \phi^{\prime} \\
& +\frac{\nu_{o}^{2}-\nu^{2}}{\nu_{o}} \int^{\phi} d \phi^{\prime} \cos \nu\left(\phi-\phi^{\prime}\right) \int^{\phi^{\prime}} f(\xi) \sin \nu_{o}\left(\phi^{\prime}-\xi\right) d \xi,
\end{aligned}
$$

where $p_{h}$ is given as $p_{h}=d q_{h} / d \phi$. The emittance function, $\epsilon$, defined as

$$
\epsilon=q^{2}+(p / \nu)^{2}
$$

is expressed using Eqs. (7) and (8) in the following way:

$$
\begin{aligned}
& \epsilon=\epsilon_{h}+\frac{2\left(\nu_{o}^{2}-\nu^{2}\right)}{\nu} \int^{\phi} \eta_{c h}\left(\phi^{\prime}\right) g\left(\phi, \phi^{\prime}\right) d \phi^{\prime} \\
&+\left(\frac{\nu_{o}^{2}-\nu^{2}}{\nu}\right)^{2} \int^{\phi} \int^{\phi} \eta_{c h}\left(\phi^{\prime}\right) \eta_{c h}(\xi) \cos \nu\left(\phi^{\prime}-\xi\right) d \phi^{\prime} d \xi \\
&+\frac{2\left(\nu_{o}^{2}-\nu^{2}\right)}{\nu_{o} \nu} \int^{\phi} g\left(\phi, \phi^{\prime}\right) d \phi^{\prime} \int^{\phi^{\prime}} f(\xi) \sin \nu_{o}\left(\phi^{\prime}-\xi\right) d \xi \\
&+\frac{2\left(\nu_{o}^{2}-\nu^{2}\right)^{2}}{\nu_{o} \nu^{2}} \int^{\phi} \int^{\phi} \eta_{c h}(\xi) \cos \nu\left(\xi-\phi^{\prime}\right) d \xi d \phi^{\prime} \int^{\phi^{\prime}} f(\sigma) \sin \nu_{o}\left(\phi^{\prime}-\sigma\right) d \sigma \\
&+\left(\frac{\nu_{o}^{2}-\nu^{2}}{\nu_{o} \nu}\right)^{2} \int^{\phi} \int^{\phi} d \xi d \xi^{\prime} \cos \nu\left(\xi-\xi^{\prime}\right) \\
& \int^{\xi} \int^{\xi^{\prime}} f(\sigma) f\left(\sigma^{\prime}\right) \sin \nu_{o}(\xi-\sigma) \sin \nu_{o}\left(\xi^{\prime}-\sigma^{\prime}\right) d \sigma d \sigma^{\prime}
\end{aligned}
$$

where $\epsilon_{h}=q_{h}^{2}+\left(p_{h} / \nu\right)^{2}$ and the function $g$ depends on the homogeneous solution in the form

$$
g\left(\phi, \phi^{\prime}\right)=q_{h}(\phi) \sin \nu\left(\phi-\phi^{\prime}\right)+p_{h}(\phi) \cos \nu\left(\phi-\phi^{\prime}\right)
$$


Assume now that the perturbation $f$ corresponds to a random process with zero average value and non-zero correlation, $K\left(\sigma-\sigma^{\prime}\right)=\left\langle f(\sigma) f\left(\sigma^{\prime}\right)\right\rangle_{r}$. In addition, if the average were taken with respect to the tune distribution, the second term on the right of Eq. (10a) would vanish. Then, the following expression can be taken for the estimation of the emittance growth variation:

$$
\begin{aligned}
\Delta \epsilon_{r}= & \left(\frac{\nu_{o}^{2}-\nu^{2}}{\nu}\right)^{2} \int^{\phi} \int^{\phi} \eta_{c h}(\xi) \eta_{c h}\left(\xi^{\prime}\right) \cos \nu\left(\xi-\xi^{\prime}\right) d \xi d \xi^{\prime} \\
+ & \left(\frac{\nu_{o}^{2}-\nu^{2}}{\nu_{o} \nu}\right)^{2} \int^{\phi} \int^{\phi} d \xi d \xi^{\prime} \cos \nu\left(\xi-\xi^{\prime}\right) \\
& \int^{\xi} \int^{\xi^{\prime}} K\left(\sigma-\sigma^{\prime}\right) \sin \nu_{o}(\xi-\sigma) \sin \nu_{o}\left(\xi^{\prime}-\sigma^{\prime}\right) d \sigma d \sigma^{\prime}
\end{aligned}
$$

where $\epsilon_{r}=\langle\epsilon\rangle_{r}$ and $\Delta \epsilon_{r}=\epsilon_{r}-\epsilon_{h}$. Consider the initial conditions of the beam given by $\eta_{c h}(0)=\eta_{0}$ and $\left(d \eta_{c h} / d \phi\right)(0)=\rho_{o}$; i.e., the homogeneous solution is given by

$$
\eta_{c h}(\phi)=\eta_{o} \cos \nu_{o} \phi+\left(\rho_{o} / \nu_{o}\right) \sin \nu_{o} \phi
$$

Consider also the case $\nu_{o}=\nu+\Delta \nu$, with $\nu_{0} \gg \Delta \nu$. Substituting these in Eq. (11) and making some rearrangements, it follows that

$$
\begin{aligned}
\Delta \epsilon_{r}= & \epsilon_{o}\left[2(1-\cos \Delta \nu \phi)+\frac{(\Delta \nu)^{2}}{2 \nu_{o}^{2}}\left(1-\cos 2 \nu_{o} \phi\right)\right]+\frac{2 \rho_{o} \eta_{o}}{\nu_{o}}(\Delta \nu \sin \Delta \nu \phi) \\
+ & \frac{4(\Delta \nu)^{2}}{\nu_{o}^{2}} \int^{\phi} \int^{\phi} d \xi d \xi^{\prime} \cos \nu\left(\xi-\xi^{\prime}\right) \\
& \int^{\xi} \int^{\xi^{\prime}} K\left(\sigma-\sigma^{\prime}\right) \sin \nu_{o}(\xi-\sigma) \sin \nu_{o}\left(\xi^{\prime}-\sigma^{\prime}\right) d \sigma d \sigma^{\prime}
\end{aligned}
$$

where $\epsilon_{o}$ is defined as $\epsilon_{o}=\eta_{o}^{2}+\left(\rho_{o} / \nu_{o}\right)^{2}$. Considering a periodic point-like random perturbation on the beam, the correlation function can be written as

$$
K\left(\sigma-\sigma^{\prime}\right)=\sum_{n, m} K_{n, m} \delta(\sigma-2 \pi n) \delta\left(\sigma^{\prime}-2 \pi m\right)
$$


Using this expression in Eq. (13), performing similar algebra as before, and effectuating the average over the tune spread within the bunch, we obtain

$$
\begin{aligned}
<\Delta \epsilon_{r}>_{\nu} & \left.=2 \epsilon_{o}\left[1-<\cos \Delta \nu \phi>_{\nu}+\frac{\left\langle(\Delta \nu)^{2}>_{\nu}\right.}{4 \nu_{o}^{2}}\left(1-\cos 2 \nu_{o} \phi\right)\right]+\frac{2 \rho_{o} \eta_{o}}{\nu_{o}}<\Delta \nu \sin \Delta \nu \phi\right\rangle_{\nu} \\
& +\frac{2}{\nu_{o}^{2}}\left[1-<\cos \Delta \nu \phi>_{\nu}+\frac{<(\Delta \nu)^{2}>_{\nu}}{4 \nu_{o}^{2}}\left(1-\cos 2 \nu_{o} \phi\right)\right] \sum_{n, m} K_{n, m} \cos 2 \pi \nu_{o}(n-m),
\end{aligned}
$$

where \langle\rangle$_{\nu}$ represents the integration over the tune distribution within the bunch. Note that for $\Delta \nu \phi \ll 1$, the first and third terms on the right side of Eq. (15) grow quadratically with the phase advance $(\phi)$, but the second term grows linearly. On the other hand, for $\Delta \nu \phi \gg 1$, the emittance oscillates around a saturation value in the form

$$
\lim _{\Delta \nu \phi \gg 1}<\Delta \epsilon_{r}>_{\nu}=2\left[1+\frac{\left\langle(\Delta \nu)^{2}>_{\nu}\right.}{4 \nu_{o}^{2}}\left(1-\cos 2 \nu_{o} \phi\right)\right]\left(\epsilon_{o}+\frac{1}{\nu_{o}^{2}} \sum_{n, m} K_{n, m} \cos 2 \pi \nu_{o}(n-m)\right) \text {. }
$$

The detailed dependence with the tune spread within the bunch depends, of course, on the tune distribution. For a uniform tune distribution,

$$
h(\Delta \nu)= \begin{cases}1 / \sigma_{\nu}, & \text { if }-\sigma_{\nu} / 2 \leq \Delta_{\nu} \leq \sigma_{\nu} / 2 \\ 0, & \text { otherwise }\end{cases}
$$

the following expressions result:

$$
\begin{gathered}
<(\Delta \nu)^{2}>_{\nu}=\sigma_{\nu}^{2} / 12 \\
<\cos \Delta \nu \phi>_{\nu}=\frac{\sin \sigma_{\nu} \phi / 2}{\sigma_{\nu} \phi / 2}
\end{gathered}
$$

and

$$
<\Delta \nu \sin \Delta \nu \phi>_{\nu}=-\frac{\cos \sigma_{\nu} \phi / 2}{\phi}+\frac{2 \sin \sigma_{\nu} \phi / 2}{\sigma_{\nu} \phi^{2}} .
$$

Therefore, the expression for the emittance growth is

$$
\begin{aligned}
<\Delta \epsilon_{r}>_{\nu}(\phi) & =2 \epsilon_{o}\left[1-\frac{\sin \sigma_{\nu} \phi / 2}{\sigma_{\nu} \phi / 2}+\frac{\sigma_{\nu}^{2}}{48 \nu_{o}^{2}}\left(1-\cos 2 \nu_{o} \phi\right)\right] \\
& +\frac{2 \rho_{o} \eta_{o}}{\nu_{o}}\left[-\frac{\cos \sigma_{\nu} \phi / 2}{\phi}+\frac{2 \sin \sigma_{\nu} \phi / 2}{\sigma_{\nu} \phi^{2}}\right] \\
& +\frac{2}{\nu_{o}^{2}}\left[1-\frac{\sin \sigma_{\nu} \phi / 2}{\sigma_{\nu} \phi / 2}+\frac{\sigma_{\nu}^{2}}{48 \nu_{o}^{2}}\left(1-\cos 2 \nu_{o} \phi\right)\right] \sum_{n, m} K_{n, m} \cos 2 \pi \nu_{o}(n-m) .
\end{aligned}
$$


The behavior of the first term between the braces is shown in Figure 1.

In practice, the tune distribution may be nonsymmetric and nonuniform. For example, the long-range beam-beam interaction can produce a nonsymmetric, nonuniform tune distribution [8].

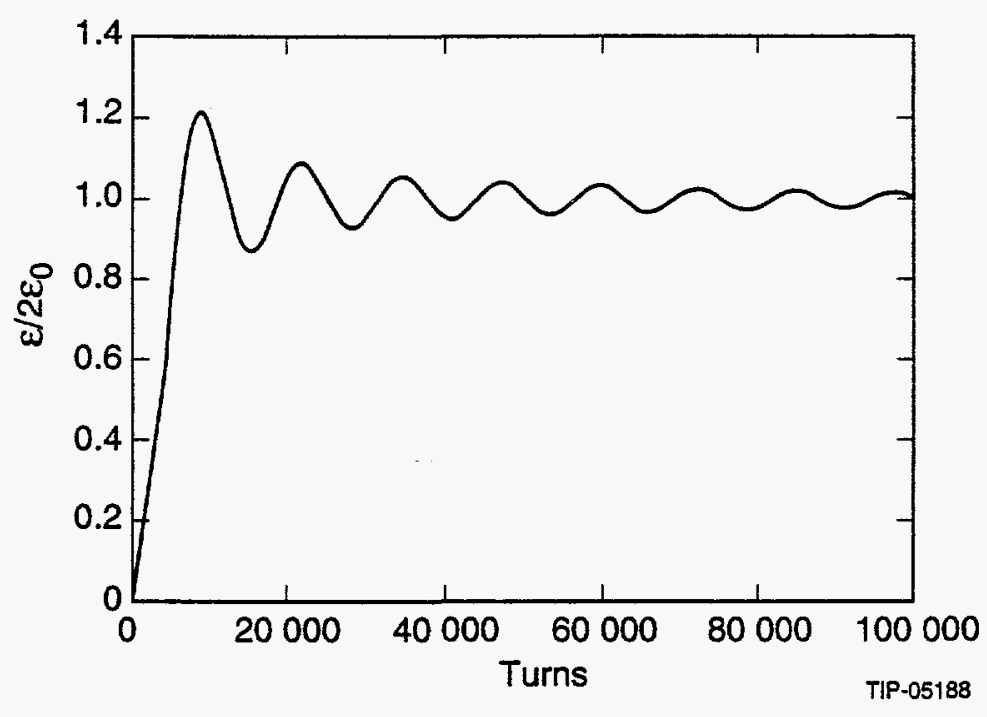

Figure 1. Saturation Effect for a Uniform Distribution.

One can notice from Eq. (13) that the above-mentioned behavior of the emittance happens even if the perturbation on the beam is uncorrelated $\left(K\left(\sigma-\sigma^{\prime}\right)=0\right)$. In this case, the emittance growth is due purely to the decoherent oscillation of the beam, i.e., $\epsilon_{o} \neq 0$. Therefore, the coherent oscillation (for example, due to injection errors) must be damped as soon as possible to avoid emittance growth. (See References 3 and 4.)

One must note from Eq. (15) that the external random perturbation may or may not be the dominant cause of emittance growth, depending on the relation between $\epsilon_{o}$ and the coefficients $K_{n, m}$. This will be demonstrated below. In the next section, a computer simulation of the emittance growth is performed for the Medium Energy Booster (MEB) of the SSC. This machine was selected due to its characteristics and the CPU time restrictions in the computer code. Our aim is not to make a quantitative check of the above calculations, but rather to verify qualitatively the results outlined above. The type of perturbation 
chosen is that of the resistive wall impedance, since the growth time of the instability is fast enough for the computer to run the program in a reasonable time.

\section{RESISTIVE WALL INSTABILITY, FEEDBACK SYSTEM,}

\section{AND EMITTANCE GROWTH SIMULATION FOR THE MEB}

The MEB is assumed to be a perfect linear machine of $3.96 \mathrm{~km}$ circumference, with an operation tune of $\nu_{o}=21.43$. This machine is filled from the Low Energy Booster (LEB) with six batches having 115 bunches each. Since the MEB injection kickers get rid of four bunches per batch, there is a total of 666 bunches, with a four-bunch gap between batches and a one-batch gap for abort proposes. However, in order to simplify the analysis, it will be assumed that 792 equally-spaced bunches $(5 \mathrm{~m})$ circulate at an energy of $11.1 \mathrm{GeV}$. Each bunch has $10^{10}$ particles with initial normalized emittance of $0.7 \mathrm{~mm}$-mrad. Although the MEB beam pipe has an elliptical shape, it will be assumed to be a cylindrical stainless steel beam pipe of radius $2.5 \mathrm{~cm}$.

The transverse resistive wall impedance is approximated as a resonant impedance (see Reference 9), where the resonant frequency is given by the revolution frequency times that fractional part of the tune between $-1 / 2$ and $1 / 2$, and the resonant impedance is given by the resistive wall impedance valuated at this resonant frequency. The growth time of the instability calculated by the computer code TADIMMI is $2.2 \mathrm{~ms}$. This instability can be seen in Figure 2, where the maximum amplitude (log scale) of the beam oscillations is plotted against the number of turns. To control this instability, a transverse feedback system was incorporated in the code. This feedback system uses a Beam Position Monitor (BPM) to measure the displacement of the bunches, and a Kicker (K) to make the correction in the trajectory of the bunches. The correction kick is given by

$$
\Delta X^{\prime}=g(X+\delta X) / \sqrt{\beta_{1} \beta_{2}},
$$

where $X$ is the displacement of the bunch, $g$ is the gain, $\delta X$ is the feedback noise (BPM resolution), and $\beta_{1}$ and $\beta_{2}$ are the Courant-Snyder beta parameters at the locations of 
the BPM and the K. The BPM and $K$ are separated by a phase advance of $\pi / 2$, and the relative separation of the resonator $(R)$ and $K$ is an arbitrary phase advance $\tilde{\phi}$. Figure 3 is a sketch of the configuration used in the simulation. To transport the 792 bunches from element to element, one uses the Courant-Snyder map. Notice that there are three perturbations affecting the bunches: the correction kick, the feedback noise, and the resistive wall impedance. Therefore, this model is much more complex than the analytical estimation discussed in Section II.

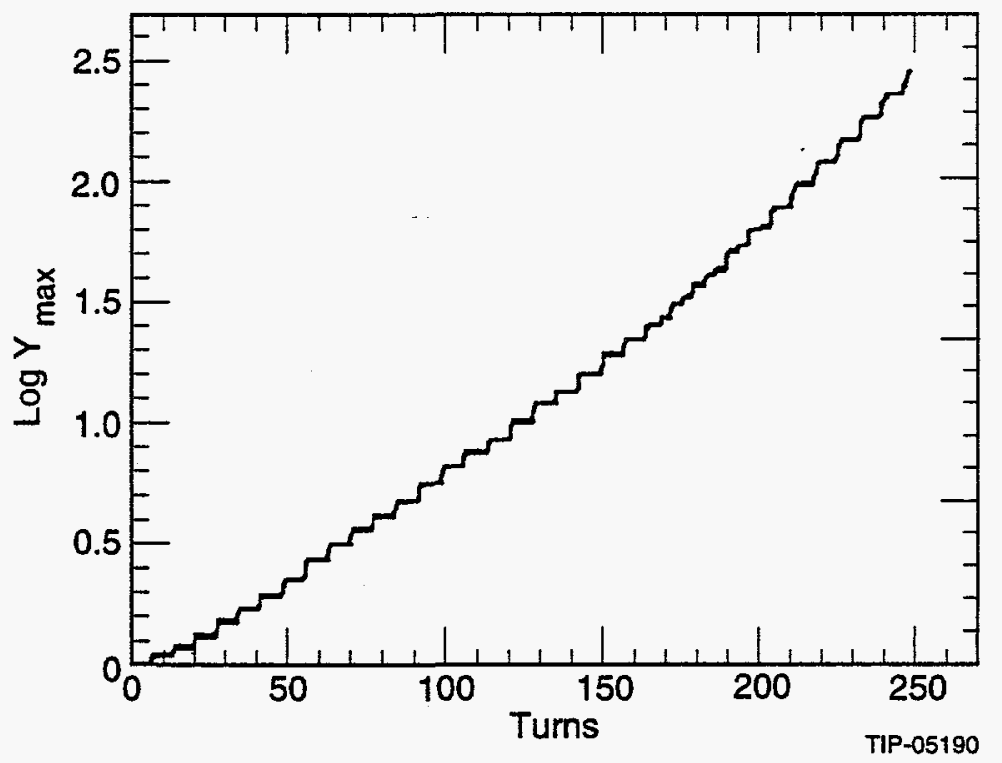

Figure 2. Growth Rate of the Dipole Mode Instability.

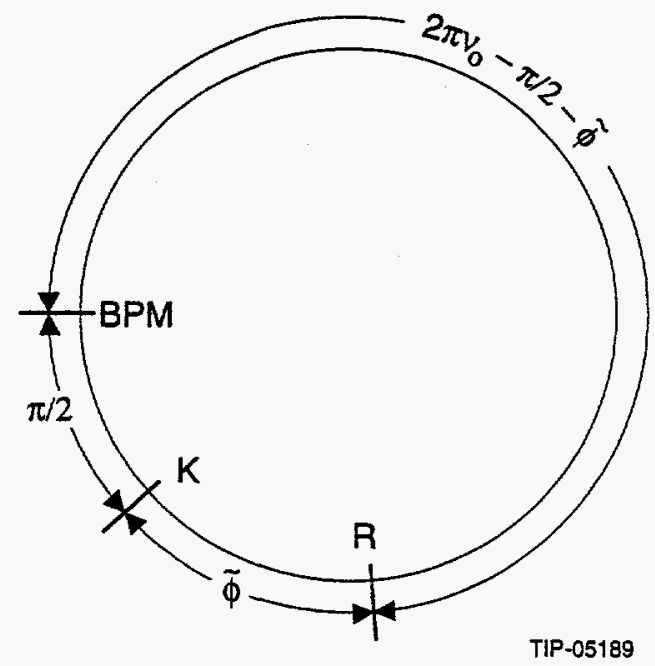

Figure 3. Sketch of the Ideal Synchrotron Machine. 
The wakefield generated at the resonator $(R)$ is that of a single-bunch macroparticle, but this electromagnetic field is felt by any submacroparticle within the bunches. Each bunch is made up of 100 submacroparticles, each of which is made up of $10^{8}$ protons. The submacroparticles have different tunes according to the assumed uniform distribution. In addition, the submacroparticles are spatially distributed within the bunch. This spatial distribution is assumed to be Gaussian, having a 1-mm sigma value (see Figure 4). To calculate the emittance associated with a particular bunch, the centroid of the bunch is calculated, $\left(X_{c}, X_{c}^{\prime}\right)$, where

$$
X_{c}=\frac{1}{N_{s}} \sum_{i=1}^{N_{s}} X_{i}
$$

and

$$
X_{c}^{\prime}=\frac{1}{N_{s}} \sum_{i=1}^{N_{s}} X_{i}^{\prime},
$$

where $N_{B}$ is the number of submacroparticles in the bunch, and $X_{i}$ and $X_{i}^{\prime}$ are the displacement and angle of the $i$ th-submacroparticle at the BPM. The Courant-Snyder emittance function is calculated through the next expression:

$$
\epsilon=\frac{1}{\beta_{1} N_{s}} \sum_{k=1}^{N_{s}}\left\{\left(X_{k}-X_{c}\right)^{2}+\left[\alpha_{1}\left(X_{k}-X_{c}\right)+\beta_{1}\left(X_{k}^{\prime}-X_{c}^{\prime}\right)\right]^{2}\right\},
$$

where $\beta_{1}$ and $\alpha_{1}$ are the Courant-Snyder parameters at the BPM location.

The bandwidth of the feedback system can be either equal to $500 \mathrm{kHz}$ (defined by the abort gap, $1.7 \mu \mathrm{s}$ ) or higher than $6 \mathrm{MHz}$ (defined by the batch-to-batch gap, $83.3 \mathrm{~ns}$ ). The minimum flat-top duration of the feedback is $26.4 \mu \mathrm{s}$ (two turns). The control of the resistive wall dipole-mode multibunch instability (at the minimum frequency) can be seen in Figures 5 and 6, where the average displacement of the bunches and the behavior of bunch 200 have been plotted as a function of the number of turns. For this simulation, a BPM resolution of $10 \mu \mathrm{m}$ and a feedback gain of 0.01 have been used.

Figures 7-10 show the evolution of the normalized emittance ratio $\left(\epsilon / \epsilon_{i}\right)$ as a function of the number of turns for the tune spreads $0.0,5 \times 10^{-4}, 0.01$, and 0.5 . Feedback characteristics such as bandwidth, gain, BPM resolution, and flat-top are the same as above. 
Figure 7 shows the oscillation of the emittance determined by the first term on the right side of Eq. (10a). Figure 8 shows the expected initial linear increase with the phase advance of the emittance, indicating that the second term on the right side of Eq. (15) is the dominant term. Figures 9 and 10 show the saturation effect in the emittance growth where the saturation value is $\epsilon / \epsilon_{i} \approx 4$ (assuming $\epsilon_{h}=\epsilon_{o}=\epsilon_{i}$ ). The expected value, according to Eq. (16), is $\epsilon / \epsilon_{i}>3$, which is in gross agreement with the simulation.

This saturation effect does not depend critically on the feedback characteristics in the resistive wall impedance case. For example, with a gain of 0.25 and a BPM resolution of $1 \mu \mathrm{m}$, the same saturation effect is found, as seen in Figures 11 and 12, where the saturation and the damping effects are shown. In other words, the feedback noise is of minor importance to the emittance growth in comparison with the decoherence and the resistive wall impedance. The resistive wall impedance kicks act as a source of strong external noise in the beam.

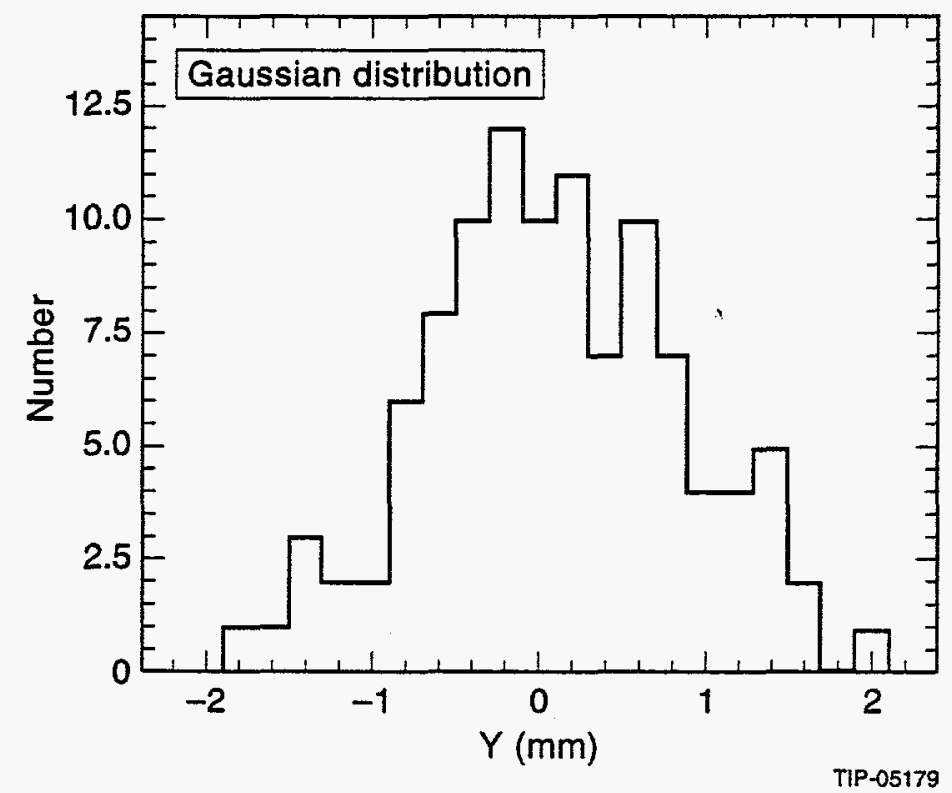

Figure 4. Approximation of a Gaussian Distribution. 


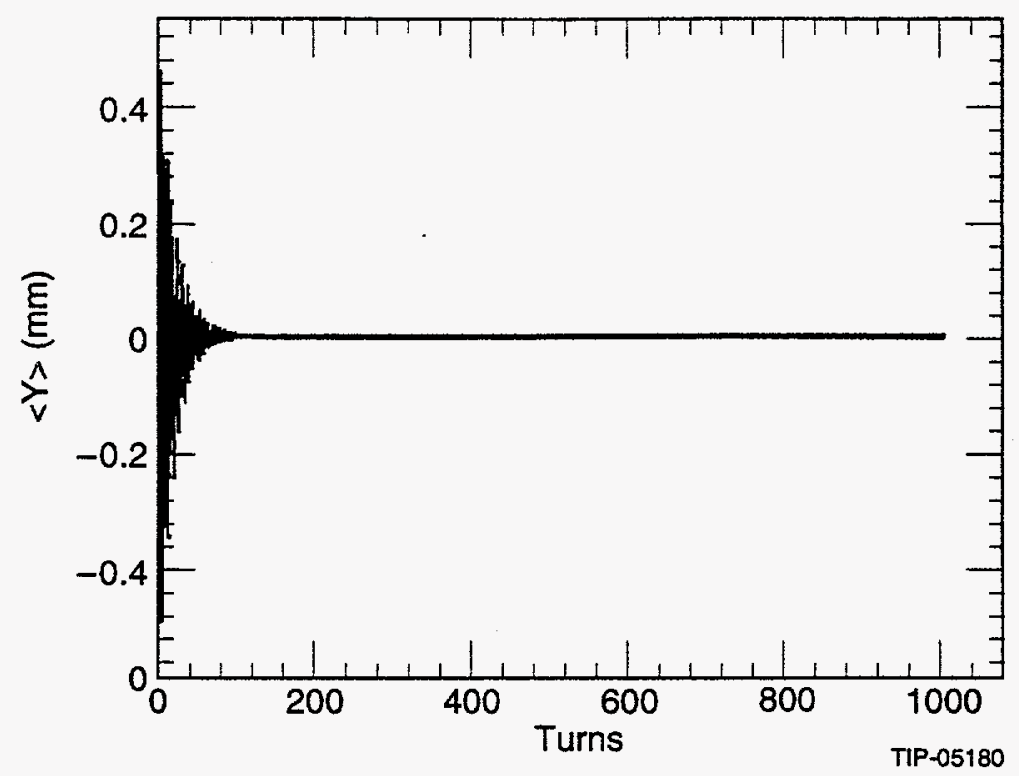

Figure 5. Damping of the Average Displacement of the Beam.

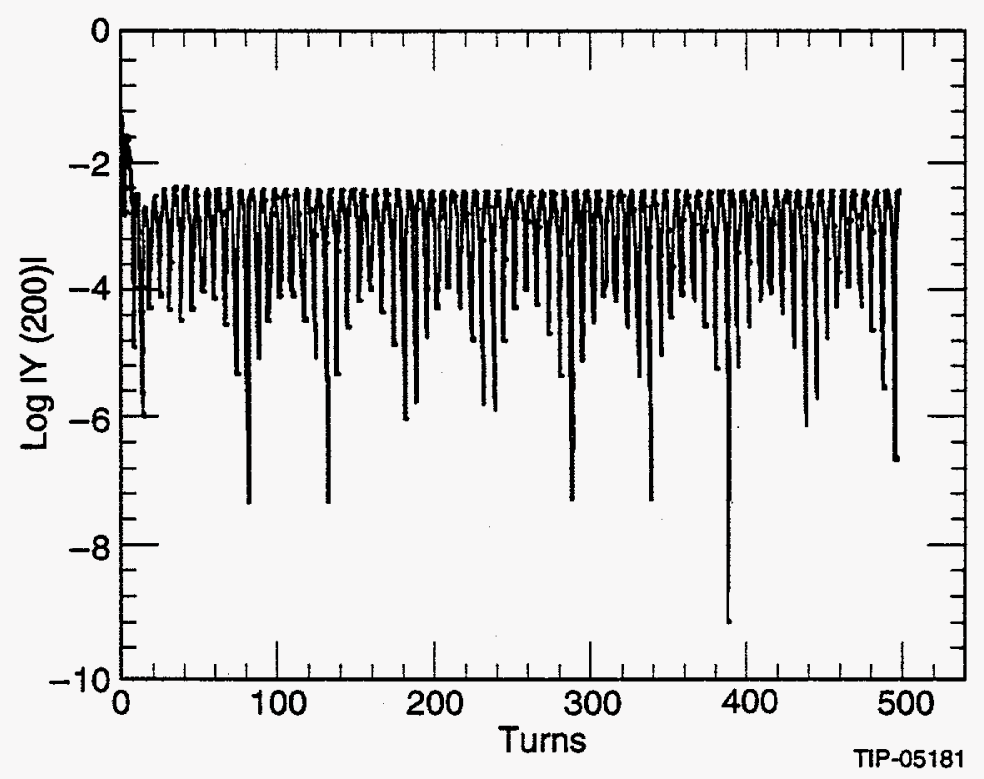

Figure 6. Damping Behavior of Bunch 200. 


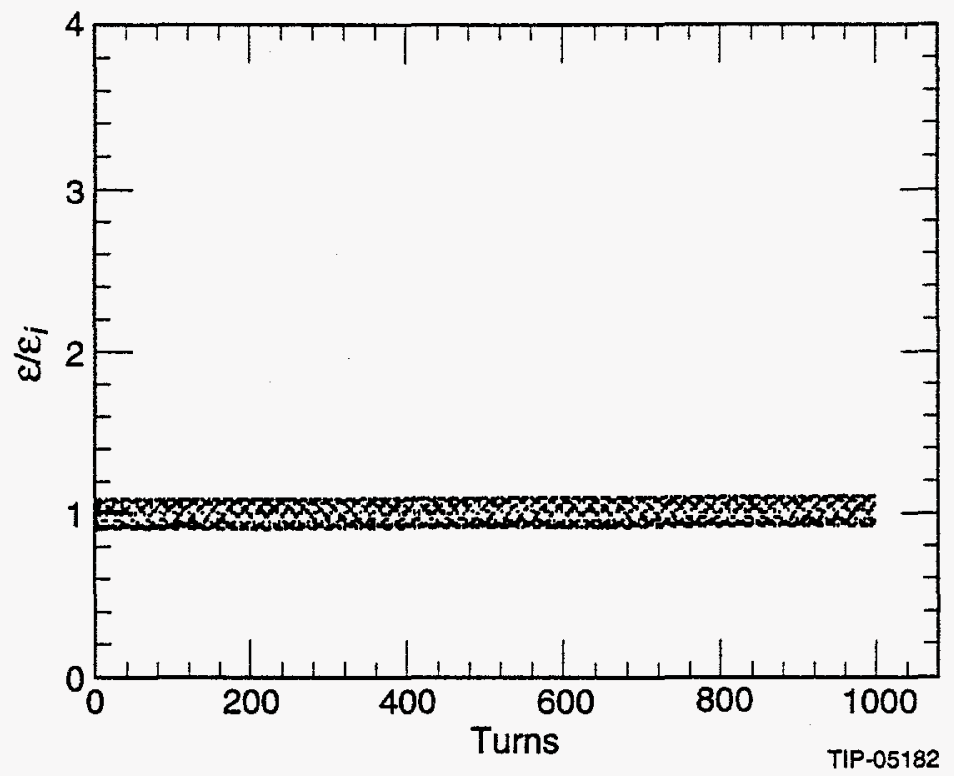

Figure 7. Emittance Oscillations $\left(\sigma_{\nu}=0.0\right)$.

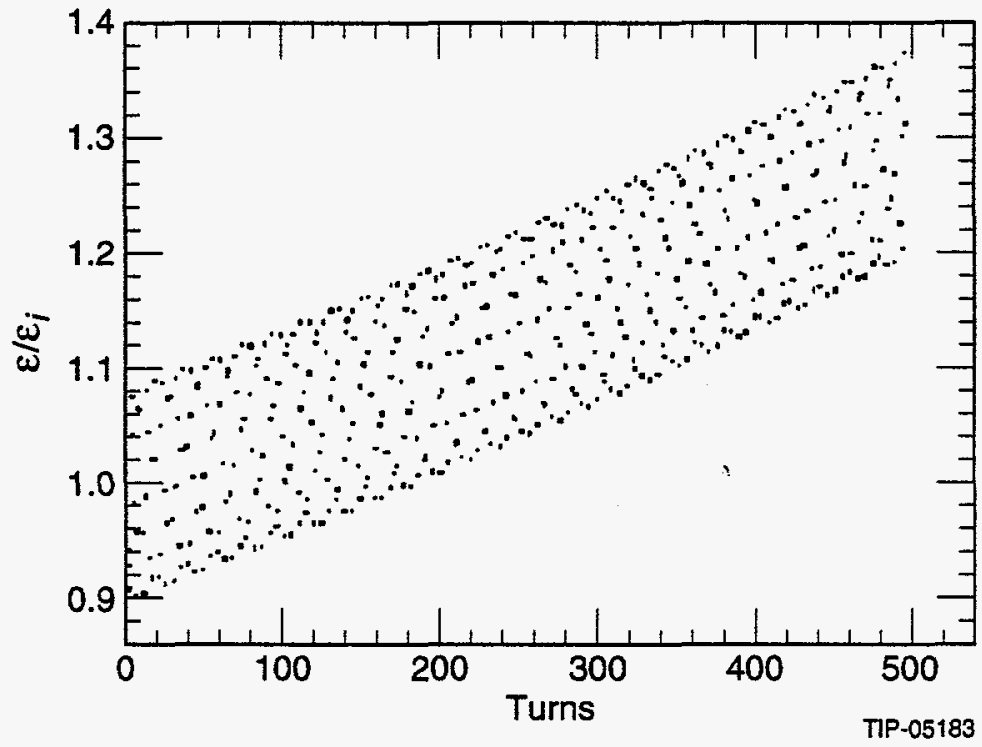

Figure 8. Initial Linear Growth of the Emittance $\left(\sigma_{\nu}=5 \times 10^{-4}\right)$. 


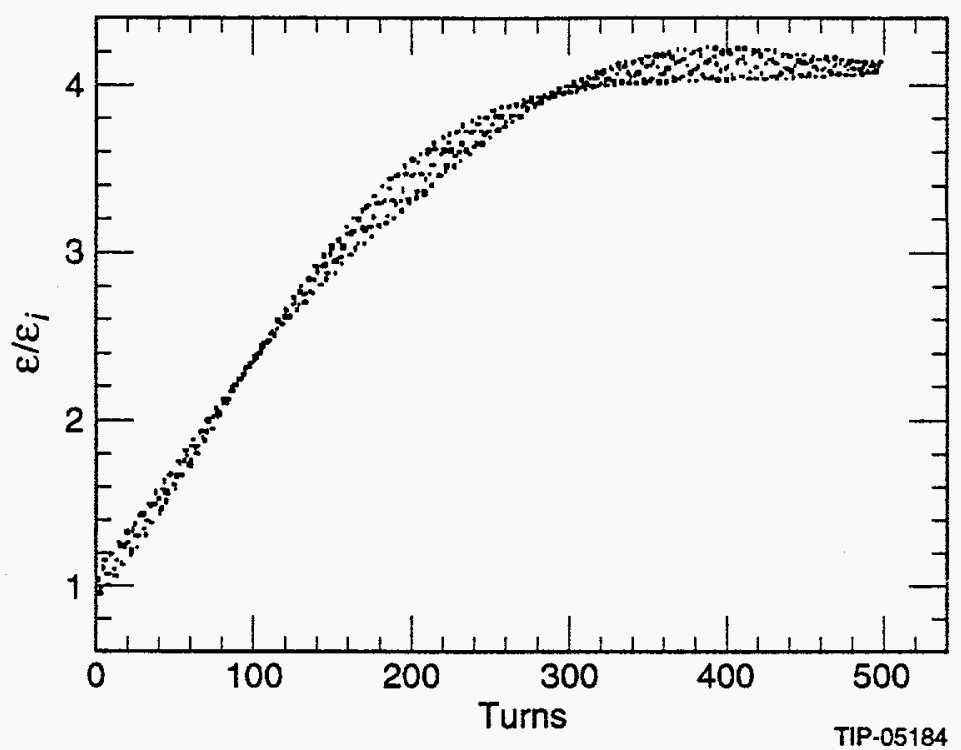

Figure 9. Emittance Growth Saturation Effect $\left(\sigma_{\nu}=0.01\right)$.

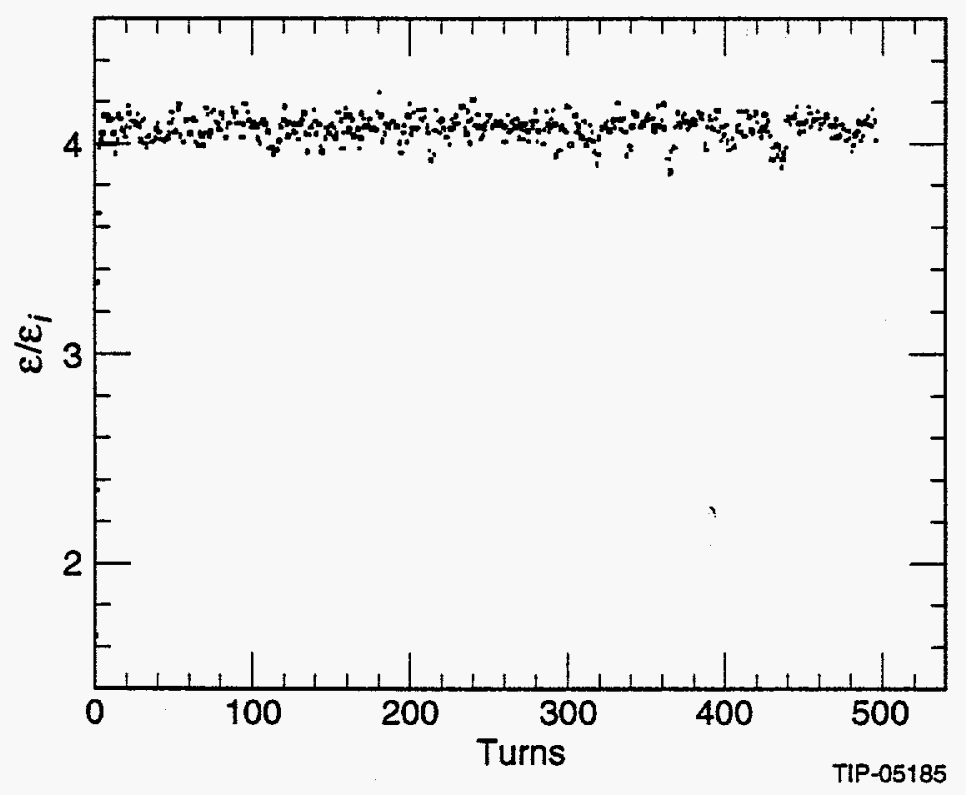

Figure 10. Emittance Growth Saturation $\left(\sigma_{\nu}=0.5\right)$. 


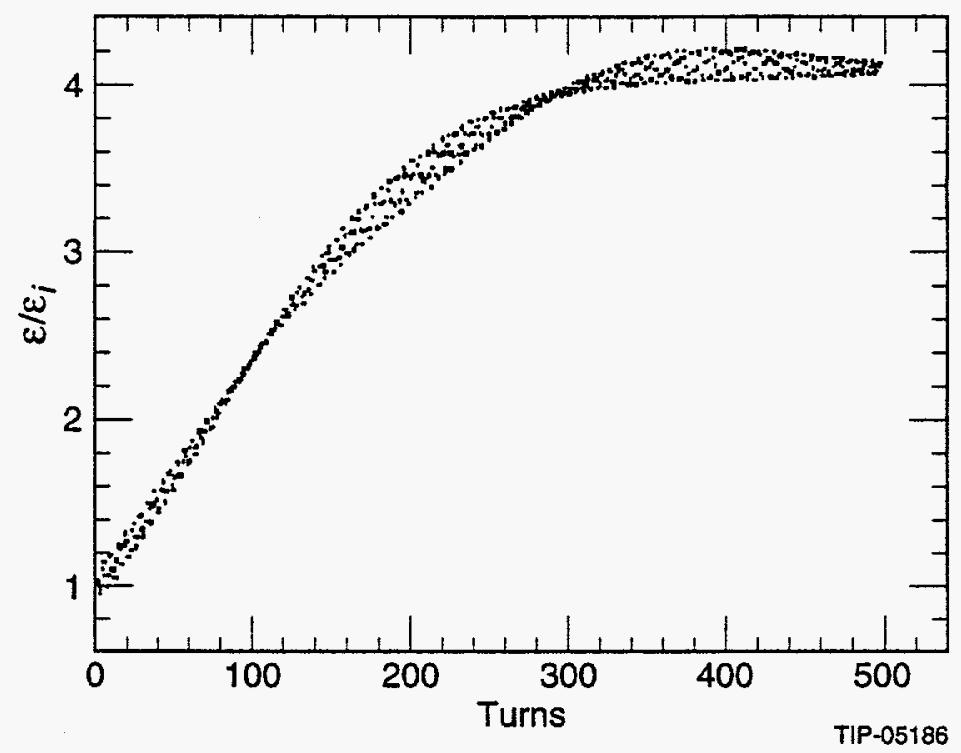

Figure 11. Saturation Effect $\left(g=0.25, \delta X=1 \mu \mathrm{m}, \sigma_{\nu}=5 \times 10^{-4}\right)$.

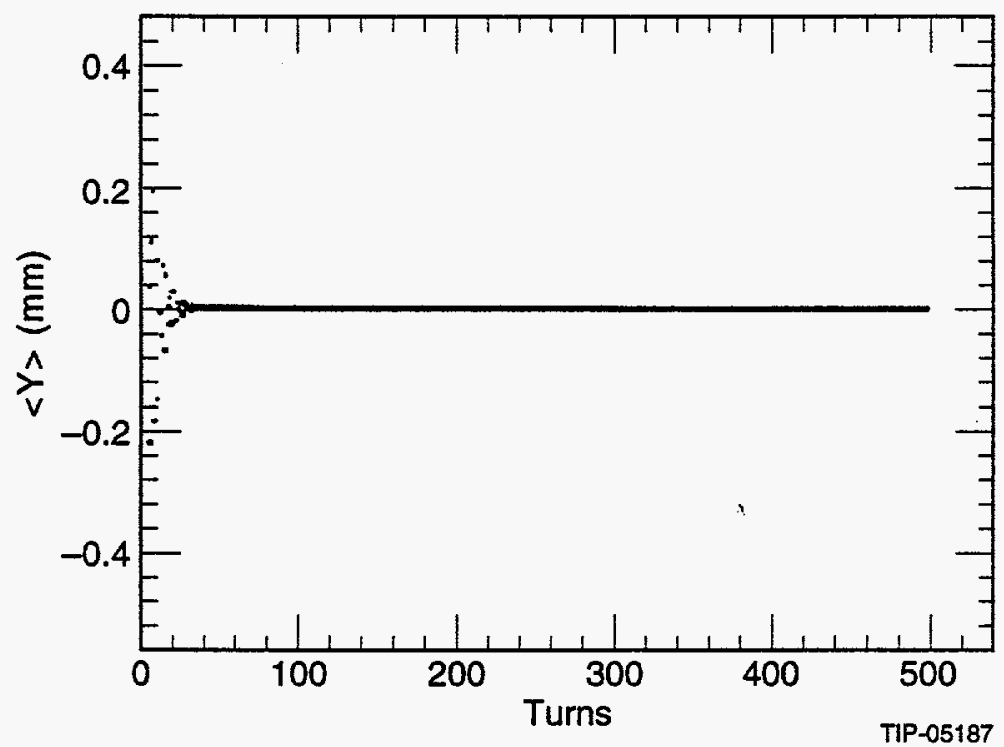

Figure 12. Damping of the Instability $(g=0.25, \delta X=1 \mu \mathrm{m})$. 


\section{CONCLUSIONS AND COMMENTS}

A single-particle analytical model was used to estimate the emittance growth in synchrotron machines due to random point-like perturbations. The calculations indicate a very strong dependence of the emittance growth on the tune spread within the bunch. The emittance growth initiates a linear increase that saturates after some time, depending on the tune spread. This qualitative behavior was confirmed with the emittance growth simulations in the MEB machine of the SSC. In summary, the analytical results obtained are consistent with those of References $3,4,10$, and 11 . In addition, it is believed that experiments can confirm this saturation effect $[12,13]$.

On the other hand, from the above studies the following picture emerges: one can design the transverse feedback system characteristics (gain, bandwidth, flat-top, and BPM resolution) to control the dipole-mode multibunch instabilities. Once those instabilities have been controlled, emittance growth depends mainly on the tune spread within the bunches. Of course, different bunches can have different tune spreads and, therefore, different growth rates. At injection, the tune spread appears due to nonlinear elements in the lattice and the nonzero chromaticity of the machine. Making the chromaticity of the machine zero and neglecting nonlinear components of the magnetic field in the machine, the tune spread can be kept to a very small value. However, if one wants to control the higher-order mode multibunch instabilities using Landau damping [14], the tune spread might be high enough such that one could expect emittance growth in the beam. One way to avoid this problem would be to use a bunch-by-bunch (high-frequency bandwidth) feedback system that could control any coherent motion within the same turn [15], and to have the smallest possible tune spread within the bunch (zero chromaticity in the machine).

\section{ACKNOWLEDGEMENTS}

We wish to thank Dr. R. Meinke and Dr. R. Gerig for their support at the SSC Laboratory. 


\section{REFERENCES}

1. "Design Study of the Large Hadron Collider (LHC)," CERN 91-03, May 1991.

2. "Site-Specific Conceptual Design," SSCL-SR-1056, July 1990.

3. V. Lebedev et al., "Emittance Growth Due to Noise and Its Suppression with the Feedback System in Large Hadron Colliders," SSCL-PP-188, March 1993.

4. G. López, "Analytical Studies of the Emittance Growth Due to the Feedback System," SSCL-625, May 1993.

5. M. Sands, "The Physics of Electron Storage Rings, An Introduction," SLAC-121, May 1979.

6. E. D. Courant and H. S. Snyder, Ann. of Phys., 3, 1 (1958).

7. E. A. Coddington, An Introduction to Ordinary Differential Equations, Prentice-Hall, Inc., 1961, p. 89.

8. G. López, "Head-On and Long-Range Beam-Beam Tune Shifts Spread in the SSC," SSCL-442, May 1991.

9. S. Chen and G. López, "Simulation Studies of the Transverse Dipole Mode Multibunch Instability for the SSC Collider," SSCL-614, January 1993.

10. L. Michelotti and F. Mills, "Amplitude growth due to random, correlated kicks," Proceedings IEEE Part. Acc. Conf., Chicago, USA, 2, 1394 (1984).

11. K. Y. Ng, "Emittance Growth due to a Small Low-Frequency Perturbation," FERMILABFN-575, November 1991.

12. W. Herr, "Diffusion of particles...," CERN SL/92-53, 1992.

13. L. Vos, "Transverse Feedback System in the CERN SPS," CERN SL/91-40, 1991.

14. G. López, "New 2BPM-1K Scheme and Resitive Wall Instability," SSCL-647, October 1993.

15. G. López et al., "Optimization of the Two Kickers Transverse Damping System for the SSC," SSCL-N-815, April 1993. 


\section{FIGURE CAPTIONS}

Figure 1. Saturation Effect for a Uniform Distribution.

Figure 2. Growth Rate of the Dipole Mode Instability.

Figure 3. Sketch of the Ideal Synchrotron Machine.

Figure 4. Approximation of a Gaussian Distribution.

Figure 5. Damping of the Average Displacement of the Beam.

Figure 6. Damping Behavior of Bunch 200.

Figure 7. Emittance Oscillations $\left(\sigma_{\nu}=0.0\right)$.

Figure 8. Initial Linear Growth of the Emittance $\left(\sigma_{\nu}=5 \times 10^{-4}\right)$.

Figure 9. Emittance Growth Saturation Effect $\left(\sigma_{\nu}=0.01\right)$.

Figure 10. Emittance Growth Saturation $\left(\sigma_{\nu}=0.5\right)$.

Figure 11. Saturation Effect $\left(g=0.25, \delta X=1 \mu \mathrm{m}, \sigma_{\nu}=5 \times 10^{-4}\right)$.

Figure 12. Damping of the Instability ( $g=0.25, \delta X=1 \mu \mathrm{m})$. 